\title{
EVIDENCE AND DIMENSIONS OF COMMERCIAL DRIVER DIFFERENTIAL CRASH RISK
}

\author{
Ronald R. Knipling \\ Truck \& Bus Safety Group \\ Virginia Tech Transportation Institute \\ Falls Church, Virginia, USA \\ E-mail: rknipling@vtti.vt.edu
}

\begin{abstract}
Summary: This paper highlights evidence from several instrumented vehicle studies that crash risk varies significantly among commercial truck drivers, and also cites findings from surveys of fleet safety managers and other experts on the topic of individual differences in commercial driver crash risk. Within various subject groups, $10-15 \%$ of the drivers typically account for $30-50 \%$ of the crash risk. This pattern is seen in measures of driver errors associated with crashes and also in measures of driver drowsiness. The evidence also suggests, but does not yet prove, that these individual differences are long-term. To the extent that these individual differences are long-term, they may be considered personal traits. This paper conceptualizes driver risk factors, provides illustrative examples of differential individual risk within groups of drivers, identifies driver factors thought to be most associated with crash risk, and considers the opportunities for improved commercial driving safety presented by differential crash risk.
\end{abstract}

\section{INTRODUCTION： TYPES OF LARGE TRUCK RISK FACTORS}

Understanding the genesis of traffic crashes requires a conceptualization of multiple interacting factors. Traditionally, contributing factors to crashes are classified as human, vehicle, and roadway/environmental; causation studies have consistently found that human causes are predominant (e.g., Treat et al., 1979; Craft and Blower, 2004). Figure 1 (Knipling et al., 2004) is a schematic of a slightly more detailed conceptualization of major crash risk factors for large trucks. Human, vehicle, and environmental factors are depicted, but the schematic explicitly includes the "4-wheeler" as a major precipitating factor. A significant percentage of large truck crashes - probably a majority-are precipitated by the actions of other drivers on the roadway. Craft and Blower (2004) have presented preliminary statistics on 287 two-vehicle crashes from the FMCSA/NHTSA Large Truck Crash Causation Study (LTCCS). In these truck-light vehicle crashes, the "critical reason" for the crash was attributed to the other vehicle or driver in $70 \%$ of the time and to the truck or truck driver in 30\%. Not all studies have found light vehicle drivers to be the principal crash precipitators, however. Council et al. (2003) examined "fault" in 199497 North Carolina police-reported truck-car crashes and reported that truck drivers were assigned "fault" in more crashes (48\%) than were car drivers (40\%).

Regardless of which driver precipitates the majority of truck-light vehicle crashes, a significant number are precipitated by the errors, misbehaviors, or other actions of large truck drivers. In Figure 1, two types of commercial driver risk factors are identified: personal situational risk factors and personal constitutional risk factors. Situational factors influencing driver alertness and fatigue include amount of prior sleep, time-of-day, and hours of continuous work. Transient 
emotional states (e.g., anger following some event) and motivations (e.g., pressure to deliver by a particular time) are also situational factors affecting driving safety.

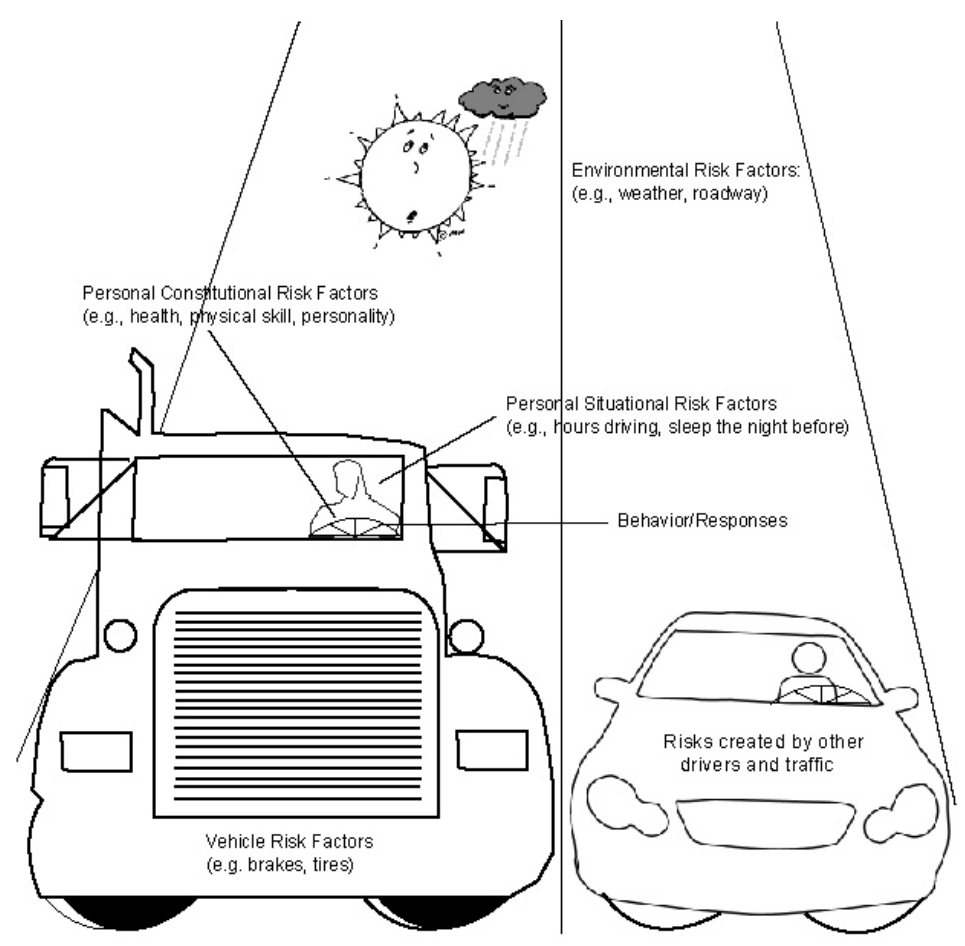

Figure 1. Interaction of factors affecting commercial driver crash involvement (Source: Knipling et al., 2004)

Recent studies (e.g., Lancaster and Ward, 2002; Knipling et al., 2004) support the view that there are also significant personal constitutional factors, or other long-term personal traits, that affect crash risk. Individual factors predisposing commercial drivers to greater or lesser risk include demographic, personality, performance, physical/medical, and behavioral history variables. This paper reviews the evidence for differential risk among commercial drivers and explores possible sources of differential risk. It is based largely on a synthesis report prepared for the Transportation Research Board (TRB) entitled, Individual Differences and the "High-Risk" Commercial Driver; A Synthesis of Safety Practice (Knipling et al., 2004).

\section{INSTRUMENTED VEHICLE STUDY EVIDENCE OF DIFFERENTIAL RISK}

Instrumented vehicle studies of commercial driving have reported wide variations in the incidence of driver error within groups of subject drivers. For example, in a naturalistic driving study involving 42 local/short haul (LSH) truck drivers, Hanowski et al. (2000) observed 249 critical incidents (CIs), which were defined as significant unsafe driver actions or "near-crashes." Of these, 77 were related primarily to the actions and errors of truck drivers. Common CIs included running late yellow or red lights and crossing traffic with insufficient gaps. The 42 truck drivers initiated 77 CIs in 1,376 hours of driving, or 0.06 truck driver-initiated CIs per hour. Figure 2 shows the frequency distribution of $\mathrm{CI} /$ hour rates among the 42 drivers. 


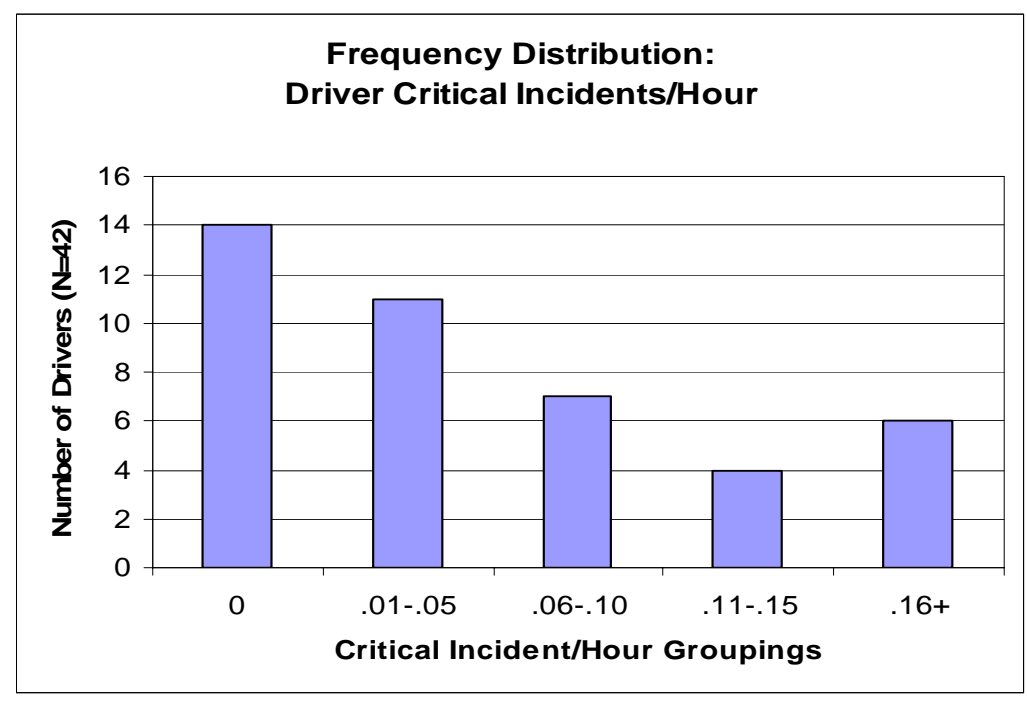

Figure 2. Frequency distribution of LSH truck driver critical incident rate (reproduced from Knipling et al. 2004, from data in Hanowski et al., 2000)

The six drivers in the far right bin of Figure 2 drove $12 \%$ of the total driving hours of the study but were responsible for $38 \%$ of all the truck driver-initiated CIs (29 of 77). In sharp contrast, the 25 "best" of the 42 drivers (the first two bins in Figure 2) drove 63\% of the driving hours but were responsible for only $16 \%$ of the CIs. In the middle (the third and fourth bins) was a moderate risk group that drove $25 \%$ of the hours and was associated with $46 \%$ of the CIs. Figure 3 illustrates these exposure-risk relationships for the three groups.

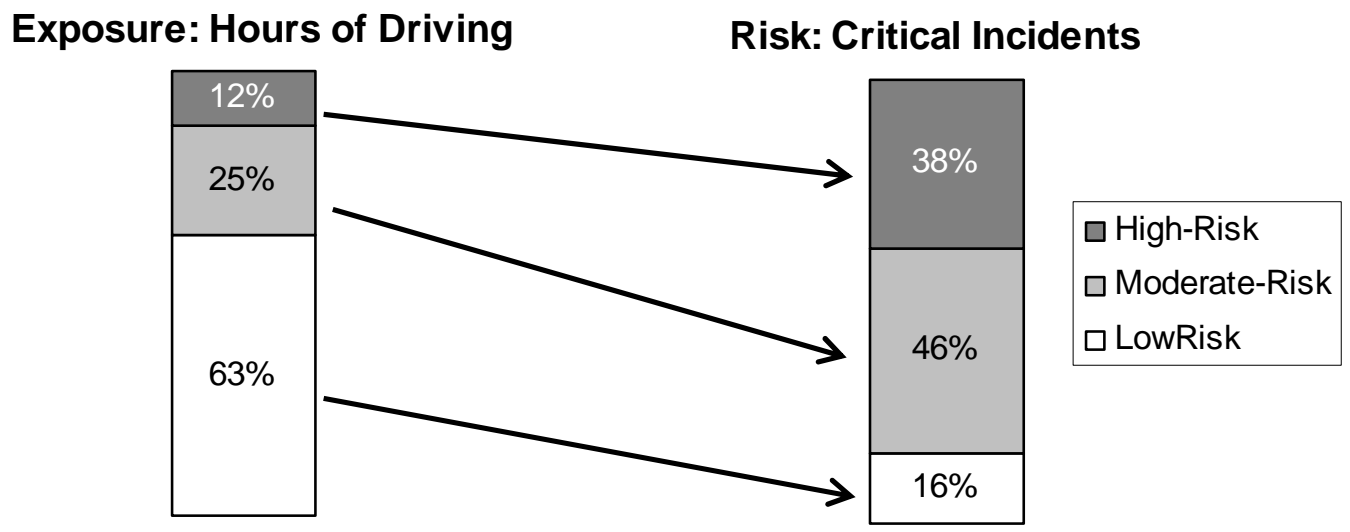

Figure 3. Relations between exposure and CI risk for high-, moderate-, and low-risk groups of drivers in the Hanowski et al. (2000) LSH truck driver study (Illustration from Knipling et al., 2004)

Similar distributions and overinvolvements of high-risk subgroups have been observed in instrumented vehicle studies that included counts of high-drowsiness episodes, including:

- Drowsiness in LSH drivers (Hanowski et al., 2000):

Highest risk drivers drove $7 \%$ of hours but had 39\% of drowsy episodes.

- Drowsiness in long-haul drivers (Wylie et al., 1996):

Eleven of 80 drivers (14\%) had 54\% of drowsy episodes. 
In a current instrumented vehicle study at VTTI, nine initial commercial driver subjects have been monitored over an average of 291 driving hours each. Dynamic triggers for identifying critical driving incidents (i.e., unsafe conditions) include high longitudinal accelerations (e.g., hard braking), short times-to-collision (close dynamic proximity to other vehicles), and hard swerves (abrupt steering). The ranges in hourly rates of occurrence of these triggers between the "best" and "worst" drivers on each scale were 67-fold for longitudinal accelerations, seven-fold for times-to-collision, and ten-fold for swerves. Intercorrelations among these three rates for the nine drivers averaged +0.52 , indicating a moderately high tendency for differential driver risk to be manifested across qualitatively different measures of risk.

Of course, not all potential confounding factors were controlled in these studies. Perhaps some drivers simply drove more difficult and dangerous routes. The biggest shortcoming of these studies in relation to a hypothesis of long-term differential driver risk is that these are all relatively short-term studies. They demonstrate wide variations in risk among individuals within participating groups of subjects, but they do not verify the existence of consistent, enduring differences nor do they reveal any underlying personal traits.

\section{SURVEY OF SAFETY MANAGERS AND OTHER EXPERTS}

In addition to the data obtained and re-analyzed from instrumented vehicle studies, a survey on the topic of individual differences and high-risk commercial drivers was conducted involving two respondent groups: 178 carrier safety managers and 67 “other experts.” The two groups averaged 22 and 18 years of experience in motor carrier safety, respectively. The two survey forms were parallel with the exception of questions on current safety practices that were relevant only to the safety managers. Both respondent samples should be characterized as convenience samples, biased toward those most interested in safety, and not statistically representative of any larger populations. Sections of the survey questionnaire addressed the importance of the problem of high-risk drivers; driver factors associated with risk; hiring, evaluation, and management practices used in fleets to address the problem; comments; and information about respondents. Highlights of the survey findings include the following:

- A majority of both safety managers (SMs) and other experts (OEs) believe that the worst $10 \%$ of drivers are associated with $50 \%$ or more of total fleet risk.

- Only 6\% of SMs and 0\% of OEs thought that risk was evenly distributed across drivers.

- When given three choices relating to the consistency of risk over time, $65 \%$ of respondents in both groups agreed that, “There is a strong tendency for drivers' risk levels to stay about the same year-to-year relative to other drivers; this year's problem driver will likely also be next year's problem driver."

- Among SMs, the five personal traits thought to be most related to driver risk (of 16 choices) were:

- Aggressive/angry

- Impatient/impulsive

- Inattentive

- Inexperienced (new CMV driver)

- Unhappy with job/company 
OE top choices were similar except that "young driver (less than 25) replaced "unhappy with job/company.”

- Among the highest-rating (for effectiveness) and most-used safety management methods relating to hiring practices were "check motor vehicle record (MVR)," on-road driving tests, alcohol/drug testing, and contacting past employers. Most of these practices are mandated by federal regulation.

- Among driver evaluation performance practices, "continuous tracking of driver crashes, incidents, and violations” was highest-rated and most-practiced.

- When asked whether rewards or discipline had a greater influence on drivers, the largest number of SM respondents rated the two approaches equal for "drivers in general," but considered discipline to have more influence on problem drivers. OE responses were similar but shifted somewhat toward rewards and away from discipline.

\section{MANAGEMENT OF “HIGH-RISK” DRIVERS}

Conceptually, there are two distinct ways that the aggregate performance of a group of drivers' safety may improve. Figure 4 illustrates two simplistic models. In the first (a), the highest-risk drivers are eliminated (e.g., they are never hired), thus "cutting off the tail” of the driver risk distribution. Even if other drivers are not affected, this intervention would have the effect of improving aggregate performance by eliminating the greatest source of risk. In the second (b), the overall distribution of safety performance of the drivers in the group is improved through some intervention. The entire curve shifts toward lower risk. Of course, the two mechanisms of improvement are not mutually exclusive and both kinds of benefits are possible through improved safety management.

The high-risk driver synthesis study conceptualized three basic management functions in relation to drivers: (1) selection and hiring, (2) performance evaluation, and (3) management of driver performance. The most obvious promising approach to improving aggregate carrier safety performance is to improve driver hiring. As one safety manager respondent put it, "Don’t hire yourself a problem ...”' Improved, systematic driver selection and hiring procedures have many potential benefits, including reduced crashes and liability most obviously, but also reduced driver turnover, increased driver job satisfaction, increased customer satisfaction, reduced personnel disputes, and increased company profitability. Many of the country's largest and most successful carriers, such as Schneider National, have highly systematic and selective driver hiring processes (Knipling et al., 2004). Unfortunately, it is not currently possible for the entire industry to be highly selective because of the chronic and worsening driver shortage. Many companies are under extreme economic and customer pressure to hire more drivers and thus be less selective in their hiring.

Once drivers are hired, what performance evaluation and management practices are likely to have the greatest impact on driver safety? Experience in other industries has shown that the most promising approaches to accident reduction focus on reducing risky behaviors. Behavior-based safety (BBS) works by identifying at-risk behaviors, observing these behaviors, measuring them, and then modifying antecedents and/or consequences to reduce them. BBS has been most successful in group settings like factories. Truck driving is usually a distributed, solitary activity, however, and so customized BBS approaches are needed. Perhaps the most promising approach 
is the use of on-board safety monitoring (OBSM) of safety-significant driving behaviors (e.g., speed, headway, longitudinal and lateral acceleration, lane tracking), combined with other BBS techniques, such as positive recognition for behavioral improvement. Progress in utilizing OBSM in long haul trucking has been slow and uneven, however, in part because of driver privacy concerns, possible adverse effects on driver retention, and perceived vulnerability to litigation following crashes. Moreover, the very worst drivers may be relatively unresponsive to reward-based BBS approaches; the most practical and prudent course of action may simply be to terminate their employment.

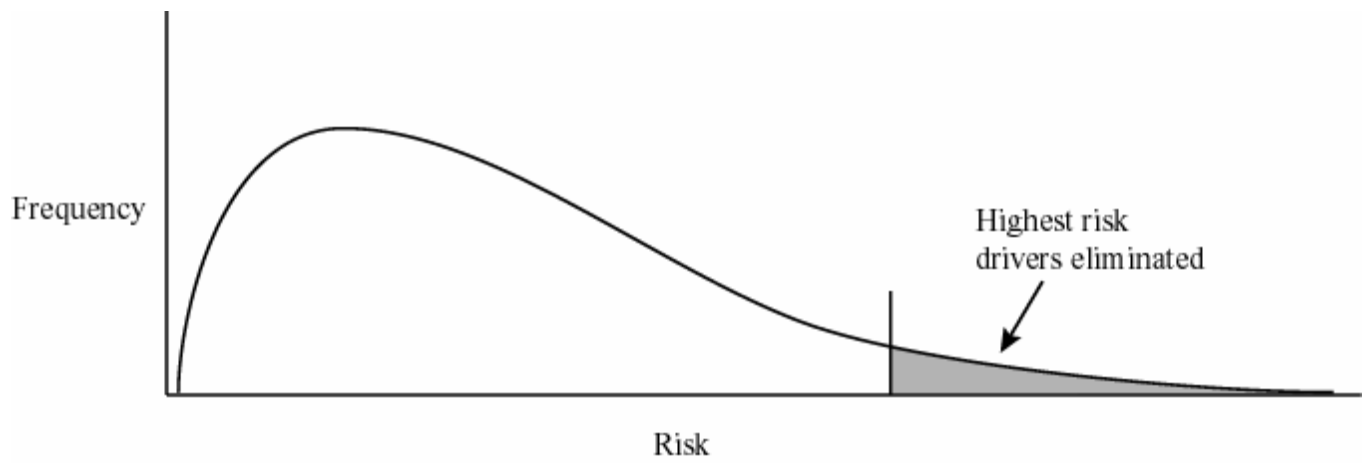

(a) “Cut off the tail” of the distribution, e.g., eliminate high-risk drivers

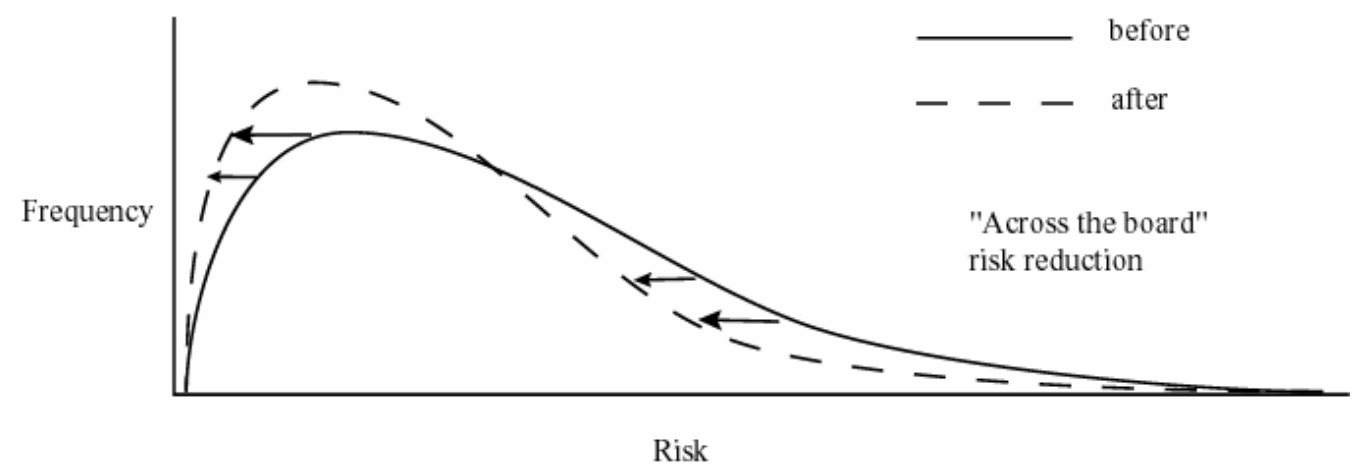

(b) Move the distribution; e.g., reduce risk for all drivers

Figure 4. Two conceptual mechanisms of improvements to a group of drivers (Illustration from Knipling et al., 2004)

\section{RESEARCH \& DEVELOPMENT (R\&D) OPPORTUNITIES}

Differential driver risk actually presents an attractive safety enhancement opportunity. If 10-15\% of drivers are consistently associated with $30-50 \%$ of risk, then safety interventions directed toward those high-risk drivers probably have far greater potential benefits than similar efforts directed toward average or better-than-average drivers. Needed R\&D to address the issue and pursue crash reduction opportunities includes, first and foremost, verifying the reliability of these provocative research findings and determining how enduring these differences are across time. To the extent that they are enduring, they constitute personal traits that may serve as the basis for 
selecting some drivers and excluding others on the basis of their probable risk. To the extent that differences are not enduring, they likely reflect situational factors or malleable behaviors.

Specific R\&D needs and opportunities identified by this study include:

- Longer-term studies to further verify the phenomenon of differential risk and determine the extent to which risk differences are consistent and enduring over time.

- The conduct of a larger case control study to profile driver individual differences within a group of drivers and associate these differences with safety outcomes (e.g., crashes, critical incidents, violations).

- Creation and field validation of improved driver selection instruments, including medical, performance, personality, and behavioral history measures.

- Research to test the proposition that differences in fatigue susceptibility are long-term personal traits, and identify efficient tests to assess level of fatigue susceptibility.

- Determination of the best safety management practices in fleets, including practices targeting problem drivers, and dissemination of this information throughout industry.

- Development and evaluation of behavioral safety management techniques, if possible enhanced by the use of on-board safety monitoring of driver safety performance and behaviors.

\section{REFERENCES}

Council, F.M., Harkey, D.L., Khattak, A.J., and Mohamedshah, Y.M. (2003). Examination of "fault," "unsafe driving acts," and "total harm" in car-truck collisions. Transportation Research Record 1830.

Craft, R. and Blower, D. (2004). The Large Truck Crash Causation Study. Paper presented and distributed at the November 17, 2004 FMCSA R\&T Stakeholder Forum, Arlington, VA.

Hanowski, R. J., Wierwille, W. W., Garness, S. A., and Dingus, T. A. (2000) Impact of Local Short Haul Operations on Driver Fatigue. FMCSA Final Report No. DOT-MC-00-203. Washington, DC: U.S. Department of Transportation.

Knipling, R.R., Boyle, L.N., Hickman, J.S., York, J.S., Daecher, C., Olsen, E.C.B., and Prailey, T.D. (2004). Synthesis \#4: Individual Differences and the High-Risk Commercial Driver. TRB Commercial Truck \& Bus Safety Synthesis Program. ISBN 0-309-08810-0.

Lancaster, R. and Ward, R. (2002). The contribution of individual factors to driving behaviour: Implications for managing work-related road safety. Entec UK Limited, Health and Safety Executive, Research Report 020, United Kingdom.

Treat, J.R., Tumbas, N.S., McDonald, S.T., Shinar, D., Hume, R.D., Mayer, R.E., Stansifer, R.L., and Catellan, N.J. (1979). Tri-Level Study of the Causes of Traffic Accidents: Final Report Volume I: Causal Factor Tabulations and Assessments. Institute for Research in Public Safety, Indiana University, DOT HS-805 085.

Wylie, C.D., Shultz, T., Miller, J.C., Mitler, M.M., and Mackie, R.R. (1996). “Commercial Motor Vehicle Driver Fatigue and Alertness Study: Project Report.” Essex Corporation. FHWA Report No. FHWA-MC-97-002. 